\title{
IL-6 blockade in systemic juvenile idiopathic arthritis - achievement of inactive disease and remission (data from the German AID-registry)
}

\author{
M. Bielak' ${ }^{1}$, E. Husmann', N. Weyandt', J.-P. Haas², B. Hügle², G. Horneff ${ }^{3,15}$, U. Neudorf' ${ }^{1}$, T. Lutz ${ }^{4}$, E. Lilienthal ${ }^{5}$, \\ T. Kallinich ${ }^{6}$, K. Tenbrock ${ }^{7}$, R. Berendes ${ }^{8}$, T. Niehues ${ }^{9}$, H. Wittkowski ${ }^{10}$, E. Weißbarth-Riedel ${ }^{11}$, G. Heubner ${ }^{12}$, \\ P. Oommen ${ }^{13}$, J. Klotsche ${ }^{14}$, Dirk Foell ${ }^{10}$ and E. Lainka ${ }^{1 *}$
}

\begin{abstract}
Background: Systemic juvenile idiopathic arthritis $(\mathrm{s} / \mathrm{A})$ is a complex disease with an autoinflammatory component of unknown etiology related to the innate immune system. A major role in the pathogenesis has been ascribed to proinflammatory cytokines like interleukin-6 (IL-6), and effective drugs inhibiting their signaling are being developed. This study evaluates sJIA patients treated with the IL-6 inhibitor tocilizumab (TCZ) concerning clinical response rate, disease course and adverse effects in a real-life clinical setting.

Methods: In 2009 a clinical and research consortium was established, including an online registry for autoinflammatory diseases (AID) (https://aid-register.de). Data for this retrospective TCZ study were documented by 13 centers.
\end{abstract}

Results: From 7/2009 to 4/2014, 200 patients with sJIA were recorded in the AID-registry. Out of these, 46 (19 m, $27 \mathrm{f}$, age 1-18 years) received therapy with TCZ. Long term treatment (median 23 months) has been documented in 24/46 patients who were evaluated according to Wallace criteria (active disease 6/24, inactive disease 5/24, remission 13/24 cases). Under observation co-medication were used in 40/46 cases. Adverse events were reported in 11/46 patients. The clinical response rate (no clinical manifestation, no increased inflammation parameters) within the first 12 weeks of treatment was calculated to be $35 \%$.

Conclusion: Out of $200 \mathrm{sJIA}$ children reported in the German AID-registry, 46 were treated with TCZ, showing a clinical response rate of 35\% during the first 12 weeks, and inactive disease and/or remission under medication in $75 \%$ after one year. Adverse events were seen in $24 \%$ and severe adverse events in $4 \%$.

Trial registration: The AID-Registry is funded by the BMBF (01GM08104, 01GM1112D, 01GM1512D).

Keywords: Systemic juvenile idiopathic arthritis, Autoinflammatory disease, Proinflammatory cytokines, Interleukin-6, Tocilizumab

\footnotetext{
* Correspondence: elke.lainka@uni-due.de

${ }^{1}$ Department of Pediatric Rheumatology, University Children's Hospital Essen,

Hufelandstr. 55, 45147 Essen, Germany

Full list of author information is available at the end of the article
} 


\section{Background}

Systemic juvenile idiopathic arthritis (sJIA) is a complex disease with an autoinflammatory component of unknown origin. sJIA is characterized by daily spiking fever which persists for at least 2 weeks, arthritis, rash, serositis, lymphadenopathy or hepato(spleno)megaly [1]. Currently, sJIA is classified by the International League of Associations for Rheumatology (ILAR) as a subtype of juvenile idiopathic arthritis (JIA) and represents about $10-20 \%$ of all JIA in the Caucasian population [2]. The ILAR classification criteria for sJIA have been criticized in the last years. Various initiatives developed new criteria for pediatric population (CARRA, PRINTO, GKJR) [3-5]. Today the most severe complication, macrophage activation syndrome (MAS), is one of the major causes of mortality in pediatric rheumatology [6]. sJIA is associated with erosive arthritis, growth retardation, cardiovascular and pulmonary morbidity as well as amyloidosis. The prevalence of amyloidosis was approximately $1-2 \%$ until the 1990s and decreased continuously in the last years [7].

sJIA is based on abnormalities in the innate immune system leading to an activation of immunocompetent cells, e.g. phagocytes, with the release of proinflammatory interleukins (ILs) like IL-1, IL-6, IL-18 and proinflammatory S100-proteins [1]. Biologicals like IL-1 inhibitors and tocilizumab (TCZ) are both approved for the treatment of sIIA [8-12]. TCZ is a humanized monoclonal antibody directed against the IL-6 receptor. It is the first biological drug being approved for the treatment of sJIA in the European Union 2011 for use alone or in combination with methotrexate (MTX), in children older than 2 years. Its effectiveness treating children younger than 2 years is under investigation [13]. By 2013, the proportion of patients with sJIA who were treated with biologicals has increased to $20 \%$ in Germany [14]. Aims: We present the first followup results of the AID-registry work concerning clinical response rates (no clinical manifestation, normal inflammation parameters), achievement of inactive disease and remission (Wallace criteria), disease courses, inflammatory parameters, outcome, concomitant medication and adverse effects from 46 out of 200 sJIA patients treated with TCZ in Germany in a real-life clinical setting.

\section{Methods}

\section{Translational AID-net}

The AID-registry is part of the AID-Net (Network for autoinflammatory diseases), a research initiative funded by the German Federal Ministry of Education and Research. Patient data are documented following a pseudonymisation procedure in an online registry (https://aidregister.de); additionally, patient material is collected and stored in a biomaterial bank for serum and plasma [15].

\section{Patients}

From 2009 to 2014, more than 200 patients with new onset or already established diagnosis of sJIA, which was confirmed by pediatric rheumatologist, were included in the AID-registry. Demographic information, clinical data, and blood samples (serum and EDTA blood) for biomarkers and genetic analysis are collected at study enrollment and follow-up visits. Generally one visit per quarter was scheduled for each patient accounting for an average of 4 visits per year in all sJIA patients. A total of 46/200 (23\%) sJIA children were treated with TCZ and longitudinally documented in follow-up. Patients treated with TCZ provided follow-up visits at week 5 and week 12 after treatment start. These patients were available for analysis of the clinical response at week 12 after start of TCZ treatment. The clinical response rate under TCZ at week 12 measured the effectiveness of TCZ and was defined as follows: no symptoms and normal inflammation parameters at that time. Remission after 12 months was evaluated based on Wallace criteria, and therefore we differentiate between active disease (AD), inactive disease (ID) and clinical remission on medication (CRM). Wallace et al. defined ID by meeting the following criteria: no active arthritis, no fever, no exanthema, no serositis, no splenomegaly, no lymphadenopathy, no active uveitis, normal ESR and CRP, no disease activity in physician's report. CRM was defined as ID for at least 6 months. Active disease (AD) described a visit with symptoms (see above) and increased inflammatory parameters [16]. CRP, ESR (after 1 and $2 \mathrm{~h}$ ) and leukocytes were measured and the number of patients with normal levels was determined. Different clinical phenotypes were defined: monocyclic (MC) means a persistent flare, after one episode the disease is inactive; polycyclic (PC) are recurrent flares (active disease changed with inactive disease); polyarticular (PA) are flares with arthritis $>4$ joints. The concomitant medication before and during TCZ application was analyzed in detail.

\section{Inclusion criteria (Fig. 1)}

We included sJIA patients who fulfilled ILAR classification criteria [17], but also other definitions for sJIA confirming diagnosis by treating pediatric rheumatologists $[3,18-20]$. For study inclusion, a minimum number of 2 visits per year were necessary for each patient. Patients fulfilling these inclusion criteria were included from the following AID-Net centers: Garmisch-Partenkirchen $(n=23)$, Essen $(n=3)$, Heidelberg $(n=3)$, St. Augustin $(\mathrm{n}=3)$, Aachen, Berlin, Bochum, Dresden, Duesseldorf, Hamburg, Krefeld, Landshut and Muenster (each $n=1-2$ ). 


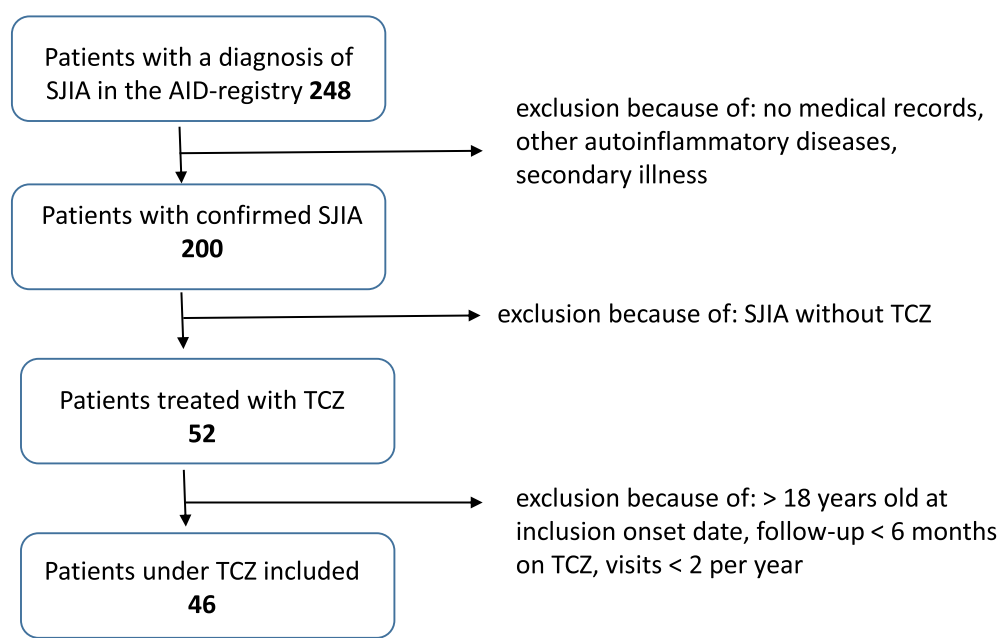

Fig. 1 Flow chart for the inclusion criteria

\section{Statistical analysis}

Descriptive analyses included mean and range for continuously distributed parameters as well as absolute and relative frequencies for categorical data. The number of adverse events was reported by 100 years under TCZ treatment. The confidence interval for the rate per 100 treatment years was estimated by exact Poisson intervals. The likelihood to achieve a clinical response under TCZ at week 12 was analyzed by logistic regression analyses. Additionally, clinical responses at various visits (response at $<5$ weeks, 6-12 weeks, 13-24 weeks, no response at $>24$ weeks) were correlated with disease activity at the last available visit in 2014. Logistic regression analyses were conducted in STATA 12.1 (StataCorp. 2011. Stata Statistical Software: Release 12. College Station, TX: StataCorp LP).

\section{Ethics commission}

The AID-registry has been approved by the ethics committees and the data protection responsibles at the University of Duisburg-Essen and Muenster, as well as the Medical Association Nordrhein in Duesseldorf. Parents, children between 9 and 13 years of age and young patients aged $\geq 14$ years provided informed consent.

\section{Results}

\section{Patient cohort}

Forty-six children $(19 \mathrm{~m}, 27 \mathrm{f})$ with a median age of 11 (range 2-21) years at their last documented visit (up to 04/2014) were included in the analyses. Age of first symptoms was 4 (range 0.6-16) years. Altogether, 680 visits were analyzed. Therapy with TCZ was started at the age of 9 (range 1-18) years. Duration from diagnosis to TCZ start was $24.5(1-188)$ months. $28 / 46$ (61\%) had a Caucasian origin (Germany, Austria, Poland, Italy, Croatia), 4/46 (9\%) were from Turkey and 6/46 (13\%) from other East European (Bulgaria, Albania) and Asian countries and 8/46 (17\%) of unknown or mixed ethnicity. 34/46 (74\%) patients fulfilled ILAR classification criteria, 12/46 (26\%) patients did not fulfill ILAR criteria but other definitions for sJIA and diagnosis was confirmed by pediatric rheumatologists [3, 18-20].

\section{Clinical and laboratory parameters}

Symptoms at diagnosis of sJIA and at the start of TCZ treatment did not differ significantly. Fever, lymphadenopathy, abdominal pain, hepatomegaly, nausea and vomiting were seen less often at TCZ start, whereas morning stiffness, myalgia, diarrhea, arthralgia and arthritis were observed more frequently. Before TCZ CRP was increased (90 (range 0.1-320) mg/l). Laboratory parameters (CRP, ESR, leukocytes) before diagnosis and under IL-6 inhibition revealed that monocyclic (MC) courses of sJIA, before initiating therapy with TCZ, showed higher CRP and ESR levels than polycyclic (PC) and polyarticular (PA) courses. After TCZ initiation CRP and ESR levels declined irrespective of the disease course. In fact, 27/46 (59\%) patients receiving TCZ showed no measurable CRP during therapy or during a clinically diagnosed flare. 10/46 (22\%) showed no measurable CRP within the first 4 weeks after starting TCZ.

\section{Response rate}

The median time under TCZ exposure was 14.9 (range 1-48) months for the 46 sJIA patients. In total, 18 (39.1\%) patients achieved an inactive disease and 14 (30.4\%) a state of remission on medication according to the Wallace criteria. Therefore, a favourable outcome under TCZ treatment was reported for 32/46 (69.6\%) of the patients in the registry. Remission on medication was additionally analyzed in a subgroup of patients who were treated with TCZ for at least 12 months. 24/46 sJIA 
patients (52\%) who received TCZ for a median of 23 (range 12-48) months were available for this analysis. The state of remission on medication was achieved for $13 / 24$ (54.2\%) sJIA patients, and additional 5/24 (20.8\%) were in a state of inactive disease, amounting 18/24 (75\%) patients with favourable outcome.

Additionally, clinical response rates within the first 12 weeks of treatment have been estimated in all 46 patients. 16/46 (35\%) patients showed an effectiveness of TCZ with no clinical manifestation and normalized inflammation parameters after 12 weeks of treatment. Comparison of initial response (at $<5$ weeks, 612 weeks, 13-24 weeks, no response at $>24$ weeks) and disease activity at last visit suggest that a rapid response to TCZ seems to be related to long term inactivity of sJIA. $7 / 17$ (41\%) patients showing inactive disease at the last visit in 2014 had a response to TCZ within 5 weeks. Active disease in the current visit in 2014 was mostly seen in patients who did not show any response within an observation time of $>24$ weeks $(8 / 12,67 \%)$.

\section{Different clinical course (Fig. 2)}

Forty-six sJIA children were categorized into monocyclic (MC) 12/46 (26\%), polycyclic (PC) 16/46 (35\%) and polyarticular (PA) 18/46 (39\%) disease courses. Comparison of these courses revealed significant differences in the outcome; PC showed the highest clinical response rate $(81 \%$, Odds ratio $(\mathrm{OR})=7.0,95 \% \mathrm{CI}: 1.8-27.2, p=$ $0.005)$ followed by the MC courses (59\%, OR $=2.9$, 95\%CI: $1.1-8.6, p=0.048)$ compared to the worst outcome for patients with PA courses (29\%).

\section{Tolerance of TCZ and non-responders (Table 1)}

Adverse events (AE) were analyzed for 46 patients contributing to $63 \mathrm{TCZ}$ exposure years. Fourteen adverse events (22.2 AE/100 TCZ exposure years, 95\%CI: 12.6-

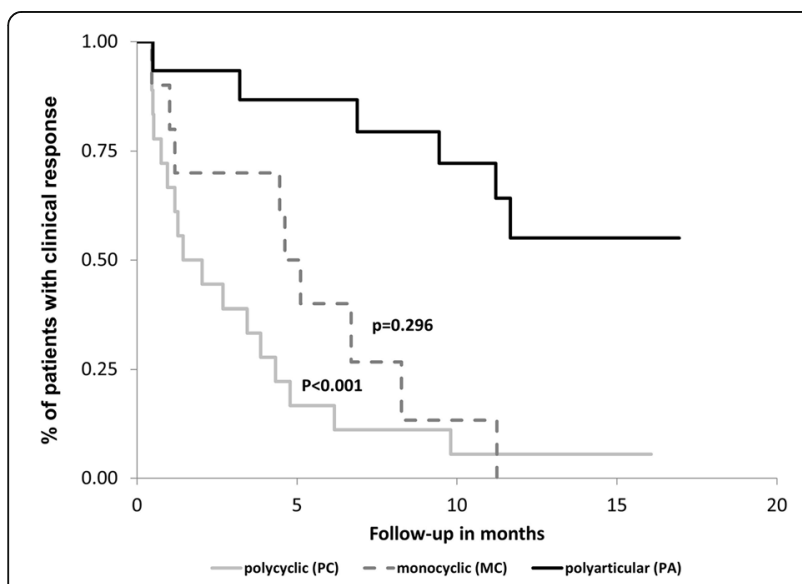

Fig. 2 Clinical response with no clinical manifestation and normalized inflammation parameters at 12 weeks of treatment by disease courses
Table 1 Adverse events (AEs) and serious adverse events (SAEs) under tocilizumab (TCZ)

AEs and SAEs $(n=16)$ under TCZ in 11/46 patients

\begin{tabular}{ll}
\hline Leukopenia & 4 \\
Recurrent infections & 3 \\
Elevated transaminases & 2 \\
Hypotonia & 1 \\
Hypertonia & 1 \\
Dyspnea & 1 \\
Abdominal pain & 1 \\
Lymphadenopathy & 1 \\
Hodgkin's-Lymphoma & 1 \\
Gut perforation & 1 \\
\hline
\end{tabular}

33.7) were reported in $11 / 46$ (24\%) patients including leukopenia, infections and elevated transaminases (at least two-fold increased), 2 serious adverse events (SAEs; 3.2 SAE/100 TCZ exposure years, 95\%CI: 0.4-11.5) were one Hodgkin's lymphoma [21] and one gut perforation [22]. No cases of MAS, amyloidosis, or death under TCZ were reported. In 2/46 MAS were diagnosed before TCZ in the medical history. In 5/46 (11\%) adverse events were the reason for stopping TCZ administration: neutropenia $n=3$, SAEs $n=2$.

Only 4/46 (9\%) patients (three with PC, one with MC disease course) had to be defined as non-responders over the whole time. In $3 / 4$ patients, symptoms got worse. They subsequently received canakinumab (CANA)/nonsteroidal anti-inflammatory drugs (NSAID), etanercept (ETA)/NSAID and anakinra (ANA)/MTX, respectively. Follow-up data from the fourth nonresponder was not available.

\section{Concomitant therapy (Table 2)}

Before starting TCZ therapy, 33/46 (72\%) patients received disease-modifying antirheumatic drugs (DMARDs) (cyclosporine A (CSA), MTX, azathioprine (AZA), abatacept (ABA), colchicine (COL)), 24/46 (52\%) biologicals (ANA, ETA, adalimumab (ADA), CANA, infliximab (IFX)), 23/46 (50\%) corticosteroids (CO) and 21/46 (46\%) NSAIDs. 6/46 (13\%) children received no co-medication before TCZ.

Co-medications at the initiation of TCZ were DMARDs (CSA, MTX, AZA) in 35/46 (76\%) patients, NSAID in $16 / 46$ (35\%) and corticosteroids (CO) in 18/ 46 (39\%) who received steroids in high- or low-dose and $3 / 18$ (17\%) who required additional steroid-pulses. 2/46 (4\%) patients received ANA or rituximab (RTX) additionally to TCZ. In our patient population 21/46 (46\%) had received TCZ as first biological treatment, 25/46 (54\%) as second, third, fourth or fifth line therapy. Among those receiving TCZ as first line treatment, 14/ 
Table 2 Co-medication before and during TCZ treatment (DMARD = CSA, MTX, AZA, ABA, COL, etc. = ANA, ETA, ADA, CANA, IFX, RTX)

\begin{tabular}{llll}
\hline Co-medication before TCZ & & Co-medication during TCZ & \\
\hline none & $6 / 46(13 \%)$ & TCZ mono & $5 / 46(11 \%)$ \\
CO mono & $5 / 46(11 \%)$ & TCZ + CO & $4 / 46(9 \%)$ \\
DMARD + etc & $15 / 46(33 \%)$ & TCZ + DMARD +etC & $22 / 46(48 \%)$ \\
DMARD + CO + etc & $18 / 46(39 \%)$ & TCZ + DMARD + CO + etc & $14 / 46(30 \%)$ \\
unknown & $2 / 46(4 \%)$ & unknown & $1 / 46(2 \%)$ \\
NSAID & $20 / 46(43 \%)$ & NSAID & $17 / 46(37 \%)$ \\
\hline
\end{tabular}

21 (67\%) showed ID or CRM after 12 months. The switch of biological agents to TCZ resulted in 14/25 (56\%) additional cases in ID or CRM. In 9/46 patients a switch from TCZ to other biological agents was seen because of non-response $(n=4)$ or adverse effects $(n=5)$.

Over the whole period of TCZ therapy corticosteroids (CO) were discontinued in 50\% (9/18) children. Under observation co-medication were used in 40/46 cases. Co-medications like DMARDs, corticosteroids (CO) and others were very variable and depended on the participating center. Monotherapy with TCZ was rare in responder ( $n=2$ and $n=1$ according to response after 12 weeks and 12 months). After 12 weeks clinical responders $(16 / 46)$ received $\mathrm{TCZ}+\mathrm{CO} n=3, \mathrm{TCZ}+$ DMARD etc. $n=11$, TCZ + DMARD etc. $+\mathrm{CO} n=0$ and only TCZ $n=2$. After 12 months clinical responders $(18 / 24)$ received $\mathrm{TCZ}+\mathrm{CO} \mathrm{n}=1, \mathrm{TCZ}+\mathrm{DMARD}$ etc. $n=15$, TCZ + DMARD etc. $+\mathrm{CO} \mathrm{n}=1$ and only TCZ $n=1$ (DMARD = CSA, MTX, AZA, ABA, COL, etc. = ANA, ETA, ADA, CANA, IFX, RTX).

\section{Discussion}

sJIA is a severe multi-organ disease of unknown etiology. Classification and diagnostic criteria are currently under discussion because sJIA is not a classical autoimmune disease like most other subtypes of JIA but more related to AID. Only $74 \%$ of the patients included into the AIDregistry fulfilled the ILAR criteria for sJIA while $26 \%$ were classified as sJIA based on other criteria [3, 17-20]. Usually sIIA is treated with various kinds of NSAIDs, and immunosuppressive drugs, but as an active disease frequently remains refractory. High-dose corticosteroids (CO) or combinations with MTX are often considered for therapy [23]. A primary aim of clinical research should be the avoidance or at least reduction of CO. Despite the availability of biological agents, one third of sJIA patients in Germany were still treated with systemic CO [14].

Long term follow-up in the AID-registry enables us to report first results on treatment, clinical response rate and tolerance of TCZ in a real-life setting. Another source of real-life data is the German Biker registry [24]. For this type of data, limitations were a small and heterogeneous population, missing data and an irregular recorded follow-up. In the past several clinical trials with TCZ have been reported for approval of TCZ in children with sJIA or withdrawal design to measure response rates during therapy with TCZ $[12,25]$. Clinical trials are generally restricted to evaluating specific intervention with a focus on assessing the efficacy and safety of therapies in a highly selected and homogeneous population. Healthcare providers often underuse treatments due to a lack of data from patients who do not meet the clinical trial inclusion criteria. While collecting data in realworld settings can be challenging, understanding the effectiveness and safety of therapies in more severe and complicated disease courses can provide reassurance for clinicians [26].

Woerner et al. describe an achievement of inactive disease with maintenance in follow-up in $37 / 77$ (48\%) patients who had received biologicals without switching. This was observed in 33/61 patients on anti-IL-1 treatment and $2 / 2$ on TCZ therapy. A switch to a second, third or fourth biological agent resulted in inactive disease in further 13/77 (17\%) patients. Median follow-up duration on biological drug was 33.8 months for patients who did not experience a switch and 6.7 months for switchers [27]. In our patient population 21/46 (46\%) had received TCZ as first biological treatment. Among those, 14/21 (67\%) showed ID or CRM after 12 months. The switch of other biological agents to TCZ resulted in ID or CRM for an additional 14/25 (56\%) cases. In 9/46 patients a switch from TCZ to other biological agents was seen because of non-response $(n=4)$ or adverse effects $(n=5)$. Kostik et al. showed that during TCZ treatment 40 out of 48 cases (83.3\%) achieved remission within 5 months. Patients who achieved remission had milder disease courses [28].

Possible courses of sJIA are monocyclic (MC), polycyclic (PC) and polyarticular (PA) (arthritis > 4 joints) activity [29]. Patients with a PA course tend to lose systemic inflammatory activity, switching to a more autoimmunelike phenotype [30]. In our cohort, 12/46 (26\%) children showed MC, 16/46 (35\%) PC and 18/46 (39\%) PA course. When applying a definition of first remission, Singh-Grewal et al. classified $42.2 \%, 20 \%$, and $37.8 \%$ of the patients as having monophasic, polycyclic, and 
persistent disease [31]. These differences are explainable by a high degree of heterogeneity in the patient population for MC and PC phenotypes. In our cohort, outcome was worst in PA courses and best in PC courses. In case of predominant polyarticular arthritis (PA) and in case of lack of response to IL-1 or IL-6 inhibition, TNFblockers were applied [5].

A large proportion of patients received MTX (89\%) and CO (66\%) before using TCZ [32]. In the second trial for approval of CANA all patients received CANA to taper steroids. In one third of the patients, $\mathrm{CO}$ could be discontinued, about half of the patients tolerated dose reduction $[1,10,11]$. With $9 / 18(50 \%)$ cases discontinuing corticosteroids under TCZ, our rate was fairly high as compared to $19 / 155$ (12\%) from the Japan cohort [26]. Oligoarticular onset, absence of active arthritis, $\mathrm{ESR}<26 \mathrm{~mm} / \mathrm{h}$ and no requirement for CO therapy at 3 and 6 months appear to be predictive of a shorter time to remission [31]. IL-1 inhibition should be considered mainly in patients with high systemic disease activity and limited joint involvement, whereas TCZ seems to be appropriate in patients with extensive joint involvement $[8,33]$. Vastert et al. started ANA in 20 patients with new-onset sJIA who were CO-naïve. At 3 months, $85 \%$ of patients achieved an adapted ACR Pedi 90 response or had inactive disease; $75 \%$ of patients achieved this response while receiving ANA monotherapy. In the majority of responding patients (73\%), treatment could be stopped within 1 year, with remission being preserved during follow-up. However, in about one third of patients, concomitant therapy was required for maintenance of clinical response [34].

These findings have led to a shift in treating sJIA, with a recent tendency to use ANA, CANA and TCZ as first line therapy [35]. Woerner et al. postulate that introducing an IL-1 or an IL-6 inhibitor as a first biologic treatment dramatically increases the chance of sJIA patients to achieve clinical remission [7]. Our results suggest that CRM correlates with a fast response to TCZ within 5 weeks of treatment. Active disease was mostly seen in patients who did not show any response within 24 weeks. There was no benefit in the outcome if TCZ was applied as first line therapy. However, challenges in the management of sJIA remain, as $30 \%$ of patients continue to present with ongoing active disease [14].

As adverse effects, nasopharyngitis, respiratory tract infections, gastroenteritis, pharyngitis and elevation of transaminases have been described previously [26, 36, 37]. In our cohort, leukocytopenia was seen most often. Kessler et al. report three cases of children with SJIA who were treated with TCZ and developed cytopenia. Two of those children had a medical history of MAS suggesting that patients with a tendency towards MAS may have an increased risk of developing cytopenia when treated with
TCZ [38]. We had two SAEs: The 15-year-old patient with multiple upper gastrointestinal perforations is published [22]. A 10-year-old girl with Becker myotonia and sJIA showed a rapid improvement of rheumatic symptoms by TCZ and steroids. After 11 months of treatment with TCZ a supraclavicular swelling was observed, histologically corresponding to lymphoma. Conceivably, at the time of sJIA diagnosis the underlying condition might already had been a Hodgkin lymphoma. As an alternative to independent occurrence of both diseases, a hypothetical causal link between TCZ and lymphoma as an AE was discussed. The Biker registry showed a risk ratio for AEs of 5.3/patient-year and for SAEs of 2.5/patient-year [24].

IL-6 induces elevation of acute phase reactants like CRP but also serum amyloid A, fibrinogen and ferritin $[39,40]$. It has a key role in the pathogenesis, clinical manifestations and activity of sJIA. This is why under IL-6 inhibition CRP levels are often not measurable and cannot be used to monitor disease activity or to detect infections [41]. 27/46 (59\%) of our patients show an active disease with CRP $<5 \mathrm{mg} / \mathrm{l}$ under therapy with $\mathrm{TCZ}$. Shimizu et al. found that serum CRP never increased during TCZ therapy, even in MAS. In contrast, serum IL-6 concentrations increased during sJIA flare-up and with the complication of infection; serum IL-18 concentrations increased before clinical disease activity. The authors suggest monitoring serum concentrations of IL-18 and IL-6 for evaluation of disease activity in SJIA and to detect the complication of infection [42]. A correlation between increase of S100 proteins and sJIA has been suggested previously [43, 44].

\section{Conclusion}

Reports on IL-6 blockade in sJIA are limited by relatively small patient numbers, diversity of study populations, highly heterogeneous treatment regimes, variable comedications, and relatively short follow-up periods. Despite these shortcomings, TCZ appears to be effective in most patients. In our real-life clinical setting, we observed a clinical response rate of $35 \%$ after 12 weeks, a remission on medication in $54 \%$ and inactive disease in $21 \%$ after 12 months. If evaluated over the entire observation time, remission on medication was achieved in $30 \%$ and inactive disease in $39 \%$ of cases. It should be noted, however, that patients often require additional treatment. Fine-tuning of dosage and co-medication will need to be addressed by future long-term studies on larger patient cohorts.

\footnotetext{
Abbreviations

AD: Active disease; ADA: Adalimumab; AE: Adverse events; AID: Autoinflammatory disease; ANA: Anakinra; AZA: Azathioprine; CANA: Canakinumab; CARRA: The Childhood Arthritis and Rheumatology Research Alliance; CO: Corticosteroids; CRM: Clinical remission under medication; CRP: C-reactive protein; CSA: Cyclosporine A; DMARDs: Disease-modifying antirheumatic drugs; DRFZ: German Rheumatism Research Centre; ESR: Erythrocyte sedimentation rate;
} 
ETA: Etanercept; GKJR: German society for paediatric rheumatology; i.v: intravenous; ID: Inactive disease; IFX: Infliximab; IL-1, IL-6, IL-18: Interleukin-1, - 6, - 18; ILAR: International League of Associations for Rheumatology; JIA: Juvenile idiopathic arthritis; MAS: Macrophage activation syndrome; MC: Monocyclic; MTX: Methotrexate; NSAIDs: Nonsteroidal anti-inflammatory drugs; p.o.: per oral; PA: Polyarticular; PC: Polycyclic; PRINTO: Pediatric Rheumatology InterNational Trials Organisation; RTX: Rituximab; s.c.: subcutaneously; SAE: Serious adverse events; sJIA: Systemic juvenile idiopathic arthritis; TCZ: Tocilizumab; TNF: Tumor necrosis factor

\section{Acknowledgements}

E. Lainka gratefully acknowledges the comments made by $O$. Weiergräber (Forschungszentrum Jülich, Germany) and thanks Volker Hilger (IT management) from the AID-registry. The authors thank all study nurses and colleagues of all participating centers for documentation in the AID-registry in Germany.

\section{Funding}

The AID-Registry is funded by the BMBF (01GM08104, 01GM1112D, 01GM1512D).

\section{Availability of data and materials}

The datasets used and/or analysed during the current study are available from the corresponding author on reasonable request.

\section{Author's contributions}

$\mathrm{MB}, \mathrm{EH}, \mathrm{NW}$ and EL analyzed data from the AID-registry and drafted the manuscript. JPH, BH, GH, UN, TL, ELi, TK, KT, RB, TN, HW, EW, GH, PO, JK, DF and $E L$ revised the manuscript critically. NW, JK and EL were responsible for statistical analyses. All authors read and approved the final manuscript.

\section{Ethics approval and consent to participate}

The AID-registry has been approved by the ethics committees and the data protection responsible at the University of Duisburg-Essen and Muenster, as well as the Medical Association Nordrhein in Duesseldorf. Parents, children between 9 and 13 years of age and young patients aged $\geq 14$ years provide informed consent. All old and new documents of the ethic committees are saved on our homepage https://www.aid-register.de.

\section{Consent for publication}

Not applicable.

\section{Competing interests}

E Lainka has received consulting fees, speaking fees, and/or honoraria from Novartis (less than $\$ 10,000$ each) and research support from Sobi. J-P Haas has received research support from Novartis and Pfizer. T Kallinich has received consulting fees, speaking fees, and/or honoraria from Bristol-MyersSquibb, CSL, Novartis and Roche (less than $\$ 10,000$ each) and research support from Novartis. E Weißbarth-Riedel has received consulting fees, speaking fees, and/or honoraria from Novartis (less than $\$ 10,000$ each). $\mathrm{H}$ Wittkowski has received consulting fees, speaking fees, and/or honoraria from Novartis (less than $\$ 10,000$ each). G Horneff has received honoraria from Abbvie, Novartis, Pfizer and Roche-Chugai (less than $\$ 10,000$ each) and research support from Abbvie, Novartis, Pfizer and Roche-Chugai. D Foell has received honoraria from Novartis, Pfizer, Roche-Chugai and Sobi (less than $\$ 10,000$ each) and research support from Novartis and Pfizer. M Bielak, E Husmann, N Weyandt, J Klotsche, E Lilienthal, T Niehues, K Tenbrock, G Heubner, T Lutz and R Berendes do not have a competing interest. U Neudorf has received speaking fees from Pfizer, Abbvie (less than $\$ 10,000$ each) und research support from Chugai (more than $\$ 10,000$ ). B Hügle has received speaking fees and/or honoraria from Novartis and Roche (less than $\$ 10,000$ each) and two unrestricted research grants from Novartis. PT Oommen has received consulting fees, speaking fees, and/or honoria from Novartis and CSL Behring (less than $\$ 5,000$ each).

\section{Publisher's Note}

Springer Nature remains neutral with regard to jurisdictional claims in published maps and institutional affiliations.

\section{Author details}

${ }^{1}$ Department of Pediatric Rheumatology, University Children's Hospital Essen, Hufelandstr. 55, 45147 Essen, Germany. ${ }^{2}$ German Center for Pediatric and
Adolescent Rheumatology, Garmisch-Partenkirchen, Germany. ${ }^{3}$ Department of Pediatrics, Asklepios Clinic Sankt Augustin, Centre for Pediatric Rheumatology, Sankt Augustin, Germany. ${ }^{4}$ Center for Pediatric and Adolescent Medicine/Pediatric Rheumatology, University Hospital Heidelberg, Heidelberg, Germany. ${ }^{5}$ Department of Pediatrics, Ruhr-University Bochum, Bochum, Germany. ${ }^{6}$ Department of Pediatric Pneumology and Immunology and Center for Chronically Sick Children of the Charité, Charité University Medicine Berlin, Berlin, Germany. ${ }^{7}$ Department of Pediatric Pneumology, Allergology and Immunology, RWTH Aachen University, Aachen, Germany. ${ }^{8}$ Department of Pediatric Rheumatology, St. Marien's Children's Hospital Landshut, Landshut, Germany. ${ }^{9}$ HELIOS Children's Hospital Krefeld, Pediatric Immunology and Rheumatology, Krefeld, Germany. ${ }^{10}$ Department of Pediatric Rheumatology and Immunology, University of Münster, Münster, Germany. ${ }^{11}$ Department of Pediatric Rheumatology, University Children's Hospital Hamburg-Eppendorf, Hamburg, Germany. ${ }^{12}$ Children's Hospital Dresden-Neustadt, Dresden, Germany. ${ }^{13}$ Department of Pediatric Oncology, Hematology and Clinical Immunology, Center for Child and Adolescent Health, Medical Faculty, Heinrich-Heine-University Düsseldorf, Düsseldorf, Germany. ${ }^{14}$ German Rheumatism Research Centre Berlin, Berlin, Germany.

${ }^{15}$ Department of Pediatric and Adolescents medicine, Medical faculty, University Hospital of Cologne, Cologne, Germany.

Received: 3 January 2018 Accepted: 9 March 2018

Published online: 05 April 2018

\section{References}

1. Bruck N, Schnabel A, Hedrich CM, et al. Current understanding of the pathophysiology of systemic juvenile idiopathic arthritis (SJIA) and targetdirected therapeutic approaches. Clin Immunol. 2015;159(1):72-83.

2. Woo P. Systemic juvenile idiopathic arthritis: diagnosis, management, and outcome. Nat Clin Pract Rheumatol. 2006;2(1):28-34. Review.

3. DeWitt EM, Kimura Y, Beukelman T, et al. Consensus treatment plans for new-onset systemic juvenile idiopathic arthritis. Arthritis Care Res (Hoboken). 2012;64(7):1001-10.

4. Martini, A. PRINTO classification for SJIA: oral presentation: toward a new classification of JIA, in PRES annual meeting. 2016: Genoa, Italy.

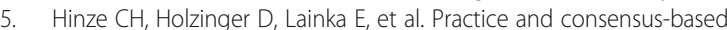
strategies in diagnosing and managing systemic juvenile idiopathic arthritis in Germany. Pediatr Rheumatol Online J. 2018;16(1):7. https://doi.org/10. 1186/s12969-018-0224-2

6. Grom AA, Horne A, De Benedetti F, et al. Macrophage activation syndrome in the era of biologic therapy. Nat Rev Rheumatol. 2016;12(5):259-68.

7. Woerner A, von Scheven-Gête A, Cimaz R, et al. Complications of systemic juvenile idiopathic arthritis: risk factors and management recommendations. Expert Rev Clin Immunol. 2015;11(5):575-88.

8. Tarp S, Amarilyo G, Foeldvari l, et al. Efficacy and safety of biological agents for systemic juvenile idiopathic arthritis: a systematic review and metaanalysis of randomized trials. Rheumatology (Oxford). 2016;55(4):669-79.

9. Yokota S, Tanaka T, Kishimoto T. Efficacy, safety and tolerability of tocilizumab in patients with systemic juvenile idiopathic arthritis. Ther Adv Musculoskelet Dis. 2012:4(6):387-97.

10. Ruperto N, Brunner $\mathrm{H}$, et al. Two randomized trials of Canakinumab in systemic juvenile idiopathic arthritis. N Engl J Med. 2012;367:2396-406.

11. Ruperto N, Quartier P, Wulffraat N, et al. A phase II, multicenter, open-label study evaluating dosing and preliminary safety and efficacy of canakinumab in systemic juvenile idiopathic arthritis with active systemic features. Arthritis Rheum. 2012;64(2):557-67.

12. De Benedetti F, Brunner HI, Ruperto N, et al. Randomized trial of tocilizumab in systemic juvenile idiopathic arthritis. N Engl J Med. 2012; 367(25):2385-95.

13. Barone P, Pignataro R, Garozzo MT, et al. IL-6 blockers in systemic onset juvenile idiopathic arthritis. Immunotherapy. 2016;8(1):79-87.

14. Klotsche J, Raab A, Niewerth M, et al. Outcome and Trends in Treatment of Systemic Juvenile Idiopathic Arthritis in the German National Pediatric Rheumatologic Database, 2000-2013. Arthritis Rheumatol. 2016;68(12):3023-34.

15. Lainka E, Bielak M, Hilger $V$, et al. Translational research network and patient registry for auto-inflammatory diseases. Rheumatology (Oxford). 2011;50(1): 237-42.

16. Wallace CA, Ruperto N, Giannini E. Preliminary criteria remission for select categories of juvenile idiopathic arthritis. J Rheumatol. 2004;31:2290-4. 
17. Petty RE, Southwood TR, Manners P, et al. International league of associations for rheumatology classification of juvenile idiopathic arthritis: second revision, Edmonton, 2001. J Rheumatol. 2004;31(2):390-2.

18. Kumar S, Kunhiraman DS, Rajam L, et al. Application of the Yamaguchi criteria for classification of "suspected" systemic juvenile idiopathic arthritis (SJIA). Pediatr Rheumatol. 2012:10:40. https://doi.org/10.1186/1546-0096-10-40.

19. Martini A. It is time to rethink juvenile idiopathic arthritis classification and nomenclature. Ann Rheum Dis. 2012;71(9):1437-9.

20. Yamaguchi M, Ohta A, Tsunematsu T, et al. Preliminary criteria for classification of adult Still's disease. J Rheumatol. 2001;19(3):424-30.

21. Forster J, Weigt-Usinger $K$, Lücke T, et al. Classical Hodgkin lymphoma in a 10-year-old girl with systemic-onset juvenile idiopathic arthritis under IL-6 inhibition (tocilizumab); 2015. https://doi.org/10.3205/15dgrh156.

22. Pfeil J, Grulich-Henn J, Wenning D, et al. Multiple upper gastrointestinal perforations in a 15-year-old patient treated with tocilizumab. Rheumatology (Oxford). 2014;53(9):1713-4.

23. Yokota S, Miyamae T, Imagawa T, et al. Clinical study of Tocilizumab in children with systemic-onset juvenile idiopathic arthritis. Clin Rev Allergy Immunol. 2005;28(3):231-8. Review.

24. Horneff G, Schulz AC, Klotsche J, et al. Experience with etanercept, tocilizumab and interleukin-1 inhibitors in systemic onset juvenile idiopathic arthritis patients from the BIKER registry. Arthritis Res Ther. 2017;19(1):256. https://doi.org/10.1186/s13075-017-1462-2.

25. Brunner HI, Ruperto N, Zuber Z, et al. Efficacy and safety of Tocilizumab in patients with polyarticular-course juvenile idiopathic arthritis: results from a phase 3, randomised, double-blind withdrawal trial. Ann Rheum Dis. 2015; 74(6):1110-7.

26. Yokota $\mathrm{S}$, Itoh $\mathrm{Y}$, Morio $T$ et al. Tocilizumab in systemic juvenile idiopathic arthritis in a real-world clinical setting: results from 1 year of postmarketing surveillance follow-up of 417 patients in Japan. Ann Rheum Dis. 2015. doi: https://doi.org/10.1136/annrheumdis-2015-207818. [Epub ahead of print]. pii: annrheumdis-2015-207818.

27. Woerner A, Uettwiller F, Melki I et al. Biological treatment in systemic juvenile idiopathic arthritis: achievement of inactive disease or clinical remission on a first, second or third biological agent. RMD Open 2015; 1(1):e000036. doi: https:/doi.org/10.1136/rmdopen-2014-000036. eCollection 2015.

28. Kostik MM, Isupova EA, Chikova IA, et al. Reasons for inactive disease and flare in systemic onset juvenile idiopathic arthritis patients during tocilizumab treatment. Clin Exp Rheumatol. 2017;

29. Haas $P$, et al. Systemic onset juvenile idiopathic arthritis - at the borderline between Autoinflammation and autoimmunity. Akt Rheumatol. 2011;36:236-41.

30. Hügle B, Hinze C, Lainka E, Fischer N, Haas JP. Development of positive antinuclear antibodies and rheumatoid factor in systemic juvenile idiopathic arthritis points toward an autoimmune phenotype later in the disease course. Pediatr Rheumatol Online J. 2014;12:28. https://doi.org/10.1186/ 1546-0096-12-28.

31. Singh-Grewal D, Schneider R, Bayer N, Feldman BM. Predictors of disease course and remission in systemic juvenile idiopathic arthritis: significance of early clinical and laboratory features. Arthritis Rheum. 2006;54(5):1595-601.

32. Kearsley-Fleet $L$, Davies $R$, Baildam E, et al. Factors associated with choice of biologic among children with juvenile idiopathic arthritis: results from two UK paediatric biologic registers. Rheumatology (Oxford). 2016;55(9):1556-65.

33. Gattorno $M$, Piccini $A$, Lasigliè $D$, et al. The pattern of response to antiinterleukin-1 treatment distinguishes two subsets of patients with systemiconset juvenile idiopathic arthritis. Arthritis Rheum. 2008;58(5):1505-15.

34. Vastert SJ, de Jager W, Noordman BJ, et al. Effectiveness of first-line treatment with recombinant interleukin-1 receptor antagonist in steroidnaive patients with new-onset systemic juvenile idiopathic arthritis: results of a prospective cohort study. Arthritis Rheumatol. 2014;66:1034-43.

35. Sikora KA, Grom AA. Update on the pathogenesis and treatment of systemic idiopathic arthritis. Curr Opin Pediatr 2011; 23(6): 640-646. doi: https://doi.org/10.1097/MOP.0b013e32834cba24. Review.

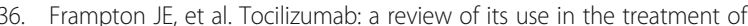
juvenile idiopathic arthritis. Paediatr Drugs. 2013;15(6):515-31.

37. Horneff $\mathrm{G}$, et al. Safety of biologic therapies for the treatment of juvenile idiopathic arthritis. Expert Opin Drug Saf. 2015;14(7):1111-26.

38. Kessler EA, Vora SS, Verbsky JW, et al. Risk of significant cytopenias after treatment with tocilizumab in systemic juvenile arthritis patients with a history of macrophage activation syndrome. Pediatr Rheumatol Online J. 2012;10(1):30.
39. Beutler B, Cerami A, et al. Cahcectin tumor necrosis factor: an endgenous mediator of shock and inflammation. Immunol. 1986:5:381-93.

40. Jain J, Gautam V, Naseem S. Acute-phase proteins: as diagnostic tool. J Pharm Bioallied Sci. 2011;3(1):118-27.

41. Opstelten W, Bijlsma JW, Gelinck LB, Hielkema CM, Verheij TJ, van Eden W. Impaired immunity: risk groups and consequences for general practice. Ned Tijdschr Geneeskd. 2016;160:A9752. Dutch

42. Shimizu M, Nakagishi Y, Kasai K, et al. Tocilizumab masks the clinical symptoms of systemic juvenile idiopathic arthritis-associated macrophage activation syndrome: the diagnostic significance of interleukin-18 and interleukin-6. Cytokine. 2012;58(2):287-94. https://doi.org/10.1016/j.cyto.2012. 02.006. Epub 2012 Mar 6

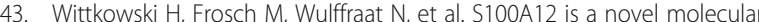
marker differentiating systemic-onset juvenile idiopathic arthritis from other causes of fever of unknown origin. Arthritis Rheum. 2008:58(12):3924-31.

44. Frosch M, Holzinger D, Roth J, et al. Systemic onset juvenile idiopathic arthritis. Monatschr Kinderheilkd. 2012;160:217-23.

\section{Submit your next manuscript to BioMed Central and we will help you at every step:}

- We accept pre-submission inquiries

- Our selector tool helps you to find the most relevant journal

- We provide round the clock customer support

- Convenient online submission

- Thorough peer review

- Inclusion in PubMed and all major indexing services

- Maximum visibility for your research

Submit your manuscript at www.biomedcentral.com/submit
Biomed Central 